\title{
Proportional Fairness in Wireless LANs and Ad Hoc Networks*
}

\author{
Li Bin Jiang and Soung Chang Liew \\ Department of Information Engineering \\ The Chinese University of Hong Kong \\ \{lbjiang3, soung\}@ie.cuhk.edu.hk
}

\begin{abstract}
This paper considers scenarios in which fairness and efficiency are two conflicting objectives in wireless networks, and investigates the use of proportional fairness objective to strike a balance between the two objectives. We explain the physical meaning of proportional fairness in a wireless network, and give an analysis showing that proportional fairness is equivalent or close to max-min fairness in terms of air-time usage (as opposed to bandwidth usage). For infrastructure WLANs, two approaches to achieving proportional fairness are discussed. For ad hoc networks, achieving proportional fairness is more complex and requires global information on contention among different traffic flows. We propose and evaluate the use of a distributed max-min air-time allocation algorithm to approximate the proportional fairness objective.
\end{abstract}

Keywords-Proportional Fairness; IEEE 802.11; WLAN; Ad hoc Networks; Distributed Algorithms

\section{INTRODUCTION}

In wireless LANs [1] and ad hoc networks [2], fairness and efficiency can be two conflicting objectives. For multirate WLANs and ad hoc networks in which nodes have the choice of transmitting at varying bit rates (e.g., in IEEE 802.11b, data rate could be $1 \mathrm{Mbps}, 2 \mathrm{Mbps}, 5.5 \mathrm{Mbps}$, or $11 \mathrm{Mbps}$ ), this issue becomes even more pronounced.

In a wireless network, a wireless station (WS) may use an "auto-rate" mechanism to choose a suitable data rate for transmission according to the channel condition experienced. Co-existing WSs could also transmit at different speeds simply because they use compatible technologies of different generations. For example, 802.11g [3] stations have a maximum data rate of $54 \mathrm{Mbps}$ while $802.11 \mathrm{~b}$ stations have a maximum data rate of $11 \mathrm{Mbps}$.

An interesting observation, as pointed out in [4] [5], is that the performance of a WLAN is usually determined by the stations with the lowest data rates. Consider a WLAN where two WSs send equal-size packets to the AP with data rates $11 \mathrm{Mbps}$ and $1 \mathrm{Mbps}$, respectively. Since the 802.11 MAC protocol implicitly provides equal access probability to both WSs, WS2 will occupy as much as 11 more air time than WS1 does, if they use similar packet sizes. As a result, the total throughput of this WLAN is only around $1 \mathrm{Mbps}$. This observation indicates that throughput fairness leads to

* This work was sponsored by the Areas of Excellence scheme established under the University Grant Committee of the Hong Kong Special Administrative Region, China (Project Number AoE/E-01/99). bandwidth underutilization in multirate wireless networks.

In fact, it is a fundamental choice whether one should strive to achieve max-min fairness (optimize "fairness"), to maximize the total throughput (maximize "efficiency"), or to strike a balance between fairness and throughput by adopting the proportional fairness [6] objective.

Inherently, the standard 802.11 multi-access protocol attempts to achieve max-min fairness in bandwidth usage. When different WSs use different data rates, the WSs transmitting at lower data rates use up more "air time". Thus, 1) max-min fairness in bandwidth usage does not translate to max-min fairness in air-time usage; (in fact, there will much unfairness in terms of air-time usage) and 2) total network throughput could be dragged down significantly when the data rates vary widely [4].

Maximizing total throughput is another extreme choice. To make the most use of the wireless medium, one could allocate the medium to WSs with the highest data rates exclusively. In this case, the low data-rate WSs will be starved and this may not be acceptable.

Proportional fairness is a compromise between fairness and throughput. Although reference [7] recommended proportional fairness as the objective of resource allocation, the corresponding physical implications for air-time usage have not been identified. Furthermore, approaches to achieve proportional fairness in ad hoc networks have not been introduced. The major contributions of this paper are 1) to show analytically that proportional fairness in bandwidth usage is equivalent or close to max-min fairness in air-time usage, and 2) to propose and investigate approaches to achieving proportional fairness in both WLANs and ad hoc networks.

\section{PHYSICAL MEANING OF PRO- PORTIONAL FAIRNESS IN MULTI-RATE WLANS}

In this section, we consider infrastructure WLANs in which all WSs communicate with an AP. As assume the AP maintains a separate queue for each WS so that for the downlink traffic, it can allocate different bandwidths to the queues in a flexible manner.

Proposition 1. Ignoring the protocol overhead such as $P H Y$ header, MAC header, backoff time, ACK, IFS, etc, proportional fairness is achieved when the fractions of air-time usage by the $W S s$ are equal. $\left(S_{i}\right.$ below includes both downlink traffic to WS $i$ and uplink traffic from WS $i$ )

Proof: 
Let $T l_{i}$ be the total amount of air-time used by WS $i$ measured over a very long period. The fraction of air-time used by WS $i$ is then

$$
r_{i}=\frac{T l_{i}}{\sum_{k=1}^{n} T l_{k}}
$$

Let $R_{i}$ be WS $i$ 's data rate. Then the throughput of WS $i, S_{i}$ is given by

$$
S_{i}=\frac{R_{i} T l_{i}}{\sum_{k} T l_{k}}
$$

For maximization of proportional fairness in the WLAN, we have Maximize $\left\{\sum_{k} \log S_{k}\right\} \quad, \quad$ or equivalently Maximize $\left\{\prod_{k} S_{k}\right\}$. Now,

$$
\prod_{i=1}^{n} S_{i}=\prod_{i=1}^{n} R_{i} * \prod_{i=1}^{n} \frac{T l_{i}}{\sum_{k=1}^{n} T l_{k}}
$$

Note that $R_{i}$ 's are constants for the optimization problem. As mentioned before, $R_{i}$ 's are pre-determined by the distances (or channel conditions) of the WSs from the AP, or by the standards $(802.11 \mathrm{~b}$ or $802.11 \mathrm{~g})$ used by their wireless cards. So, Maximize $\left\{\prod_{k} S_{k}\right\}$ is equivalent to maximizing $\prod_{i=1}^{n} \frac{T l_{i}}{\sum_{k=1}^{n} T l_{k}}$.

$$
\begin{gathered}
\text { Since } \sum_{i=1}^{n} \frac{T l_{i}}{\sum_{k=1}^{n} T l_{k}}=1, \prod_{i=1}^{n} S_{i} \text { is maximized when } \\
T l_{1}=T l_{2}=\ldots=T l_{n}
\end{gathered}
$$

From (1) and (4), we have

$$
r_{1}=r_{2}=\ldots=r_{n}=1 / n
$$

completing the proof of the proposition.

If (5) is satisfied, then

$$
S_{i}=\frac{R_{i} T l_{i}}{\sum_{k} T l_{k}}=\frac{R_{i}}{n}
$$

This is an interesting property of proportional fairness in WLANs.

Property 1. In a WLAN, given a fixed number of WSs, the throughput of one WS is independent of the data rates used by other WSs, if proportional fairness is achieved.

We notice that in [8], a similar notion of "Time-based Fairness" is proposed to achieve higher efficiency and reasonable fairness in multi-rate WLANs. We have shown here, however, that "air-time fairness" is a natural result of the more fundamental "proportional fairness". Also, [8] does not take into account the protocol overhead (which we consider in Proposition 2).

Proposition 1 and Property 1 are general for any network with the same medium sharing model. (As a special case, when all the WSs use the same data rate, proportional fairness reduces to max-min fairness in terms of throughput.)

The observation in Proposition 1 motivates us to conduct a more detailed investigation to take into account the protocol overhead in WLANs. For an analysis, let us first define the terms "slots" and "Tx Ratio". If there are always packets to be transmitted in a WLAN (Saturation), wee can divide time into "slots", and there are two kinds of "slots" [10], as shown in Fig 1 .

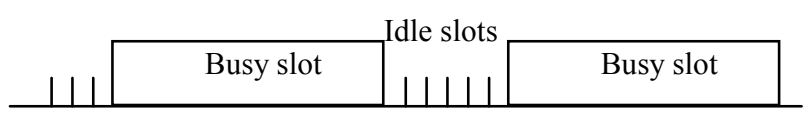

Figure 1. Idle slots and Busy slots

(a). Idle slot: $\sigma$ (e.g., $20 \mu s$ in $802.11 \mathrm{~b}$ while $9 \mu \mathrm{s}$ in 802.11a) for counting down without transmission. Before transmitting a packet, a station needs to wait for $k$ idle slots, where $k$ is randomly chosen in a Contention Window [0, $W-1]$.

(b). Busy slot: The transmission interval $T s$ (successful) or $T f$ (unsuccessful).

$T s$ is the time required for a successful packet (or "packet burst", explained in Section III) transmission, and Tf is the time wasted for an unsuccessful transmission, which could be caused by either a packet collision or channel errors. $T s$ and $T f$ are determined by the 802.11 specifications of data rates, PHY header, MAC header, ACK, DIFS, SIFS, and the length of the payload [10]. If we do not consider packet burst, we have

$$
\begin{gathered}
T s=t_{P H Y_{-} \text {header }}+t_{M_{A C_{-} \text {header }}}+t_{p}+S I F S+\delta+t_{A C K}+D I F S+\delta(7) \\
T f=t_{P H Y_{-} \text {header }}+t_{M A C_{-} \text {header }}+t_{p}+D I F S+\delta
\end{gathered}
$$

where $t_{p}$ is the transmission time needed for the MAC layer payload, and $\delta$ is propagation delay. In the following, we will add a subscript $i$ to denote the parameters for a packet targeted for WS $i$.

Tx Ratio (Transmission Ratio, denoted as $\tau$ ) of one WS is defined as the ratio of the number of busy slots (including both successful and unsuccessful busy slots) occupied by this WS to the total number of idle slots. Note that this definition is different from the "transmission probability" in [10]. If we consider a scenario with only downlink traffic from the AP to its $n$ WSs, it is clear that

$$
\tau_{0}=\sum_{i=1}^{n} \tau_{i}
$$

where $\tau_{0}$ is the Tx Ratio of the AP as a whole.

Proposition 2. Considering the protocol overhead, if there is only downlink traffic in an infrastructure WLAN, proportional fairness is achieved when all the WSs in the WLAN have the same fraction of "effective air-time usage", defined as follows:

$$
r_{e_{i}}=\frac{\tau_{i} T_{i}}{\sum_{j=1}^{n} \tau_{j} T_{j}}
$$

where $\tau_{i}$ is the Tx ratio of WS $i$, and $T_{i}$ is the effective transmission time of one packet targeted for WS $i$ : 


$$
T_{i}=\bar{T}_{i}+\frac{\sigma}{\sum_{j=1}^{n} \tau_{j}}=\bar{T}_{i}+\frac{\sigma}{\tau_{0}}
$$

where $\bar{T}_{i}$ is the transmission time for the payload to WS i plus all the protocol overhead (PHY header, MAC header, ACK, DIFS and SIFS). Given the same packet size, the lower the data rate, the larger the value of $\bar{T}_{i}$. If no channel errors are assumed, $\bar{T}_{i}=T s_{i}$, as in Equation (7). $\frac{1}{\tau_{0}}$ can be regarded as the average number of idle slots per packet.

Note: In practice when most traffic in a WLAN is downlink TCP_DATA flows (TCP_ACK packets are very small and fewer by comparison), pure downlink traffic may be a good approximation.

Proof:

Recall equation (9), $\tau_{0}=\sum_{i=1}^{n} \tau_{i}$, Note that $\tau_{0}=\frac{2}{W-1}$, where $\mathrm{W}$ is the initial contention window ( $W=C W M i n$, with a default value 32 in 802.11 b) of the AP.

We define the "effective data rate" $R e_{i}$ as

$$
R e_{i}=\frac{P_{i}}{T_{i}}
$$

where $P i$ is the average packet size for packets to WS $i$. Then the throughput of each flow can be expressed as

$$
S_{i}=\frac{\tau_{i} P_{i}}{\sum_{j=1}^{n} \tau_{j} T_{j}}=\frac{\operatorname{Re}_{i} \tau_{i} T_{i}}{\sum_{j=1}^{n} \tau_{j} T_{j}}
$$

Notice that Equation (13) has the same structure as Equation (2). So the same conclusion as Proposition 1 follows here. That is,

$$
\begin{aligned}
& \prod_{j=1}^{n} S_{j} \text { is maximized when } \\
& \qquad \tau_{1} T_{1}=\tau_{2} T_{2}=\ldots=\tau_{n} T_{n}
\end{aligned}
$$

From (10) and (14), we have

$$
r_{e_{1}}=r_{e_{2}}=\ldots=r_{e_{n}}=1 / n
$$

completing the proof of the proposition.

Conjecture: Considering both uplink and downlink traffic in a WLAN, although packet collisions make the closed-form expression of individual throughput difficult to obtain, we could approximate this situation as in Proposition 1. So, we conjecture Proposition 1 also holds on an approximated basis.

We will validate this conjecture by simulation in Section $\mathrm{V}$.

\section{IMPLEMENTATION OF PROPORTIONAL FAIRNESS IN A WLAN}

In a WLAN, all the WSs are in the range of AP. For downlink traffic, the AP acts as the coordinator to allocate the air-time equally to the WSs. The "air-time" includes the time for PHY header, MAC header, ACK, SIFS, DIFS as in
Proposition 2. (The time for idle slots is neglected.) For uplink traffic, there are two approaches.

\section{A. Using equal TXOP length in each WS}

A straightforward approach to implement proportional fairness for uplink traffic in a WLAN is to utilize packet bursting from WSs, as defined in 802.11e standard [11], while keeping the CWs unchanged (identical for all the WSs). The bursting lengths (i.e., TXOP lengths, expressed in "seconds") are set as the same for all WSs to achieve equal air-time usage, independent of the WSs' individual data rates. Since 802.11 MAC implicitly provides long-term equal access probability to all the WSs, equal TXOP for all the WSs leads to equal airtime occupancy in a long term.

\section{B. Adjusting Initial CWs of different WSs}

We can alternatively tune the initial CWs (CWMin's) of the WSs to achieve this goal for uplink traffic. Considering the overhead, assuming saturated uplink traffic,

Let $W_{i}$ be the initial CW (CWMin) of WS $i$. Tx Ratio of WS $i$ can be expressed as (readily derived from "transmission probability" [10])

$$
\tau_{i}=\frac{2\left(1-2 p_{i}\right)}{\left(1-2 p_{i}\right)\left(W_{i}-1\right)+p_{i} W_{i}\left(1-\left(2 p_{i}\right)^{m}\right)}
$$

where $p_{i}$ 's refer to the collision probability experienced by WS $i$ 's uplink flow. An uplink flow experiences collisions with downlink packets, as well as with other uplink packets. But when the number of stations is large, and $\tau_{i}$ 's are small, the collisions seen by one WS is close to those seen by another WS. As a result, $p_{i}$ 's should be nearly the same for all the WSs. If $W_{i}$ 's are large enough, we have

$$
\tau_{1}: \tau_{2}: \ldots: \tau_{n} \approx \frac{1}{W_{1}}: \frac{1}{W_{2}}: \ldots: \frac{1}{W_{n}}
$$

Then, to achieve time fairness (14), we could simply let

$$
W_{i} \propto T_{i}
$$

where $T_{i}$ here is the air time including the protocol overhead in one burst (containing one or multiple packets) used by WS $i$. The value of CWMin's can be distributed from the AP to the WSs, since the AP knows the individual air-time used by one burst from each WS.

\section{PROPORTIONAL FAIRNESS IN AD HOC NETWORKS}

Nodes in ad hoc networks can also be multi-rate. As mentioned before, max-min fairness is not efficient in this situation, proportional fairness may be preferable. Achieving proportional fairness in ad hoc networks is more challenging, due to its distributed nature.

To formulate the problem, let us first briefly review the conception of flow contention graph and clique [9]. Let $N$ be the number of flows in an ad hoc network, these flows constitute a set $F$. ("flow" here means "MAC layer flow", i.e., one-hop.) The flow contention graph of $F, G(F)$ contains the interfering relationships among the $N$ flows. (Two examples will be given in Fig 3 and Fig 5 (a).) A vertex in this graph represents a flow, and an edge between two vertices indicates that these two flows can not exist simultaneously (i.e. they 
interfere with each other). In [9], for example, two flows mutually interferes if

$$
\begin{aligned}
& |T a-T b|<C S \text {, or } \\
& |T a-R b|<C S \text {, or } \\
& |R a-T b|<C S \text {, or } \\
& |R a-R b|<C S
\end{aligned}
$$

where $T a, R a, T b, R b$ refer to the transmitter and receiver of flow a and flow b. $C S$ refer to Carrier Sense Range. In different interfering models and multi-access protocols, the actual interference range and inequalities describing the conditions may be different. A more elaborate model can be found in [12].

A clique $C l$, is a subset in $F$ where each pair of flows in this subset are interfering with each other. In Fig 3 (b), for example, $\{1,2,3,4\}$ and $\{4,5,6\}$ are two cliques.

The following optimization problem needs to be solved to achieve proportional fairness in an Ad hoc network:

$$
\begin{aligned}
& \operatorname{Max}\left\{\prod_{j=1}^{N} r_{j} R_{j}\right\}, \\
& \text { subject to } r_{i} \geq 0, i=1,2, \ldots, N \\
& \text { and } \sum_{j \in l_{k}} r_{j} \leq 1, k=1,2, \ldots, m
\end{aligned}
$$

where $R_{j}$ is the data rate of flow $j, C l_{k}$ is a clique of the flow contention graph [9] where in total $m$ cliques exist, $r_{i}$ is the fraction of air time used by flow $i$.

According to (19), each WS needs global information to compute its fair share in terms of time usage. This is difficult in a distributed network without a coordinator. But, recall that bandwidth proportional fairness in a WLAN is equivalent to air-time max-min fairness. So, we propose to allocate the share of air-time $r_{j}$ to node $j$ in a max-min manner based on the situation in its neighborhood rather than the whole network, in order to approximate proportional fairness.

In [9], a set of distributed algorithms has been developed to achieve max-min fairness of individual throughput. Only minor modifications are needed to meet our objective. The modified set of algorithms are called "Time-Max-Min" algorithm. First, each WS with packets to send computes a "fair share" of "air time" it should get, according to information of its neighbors, instead of the whole network (Algorithm 1 [9]). Then, a WS in the network needs to measure the "air time" (in seconds, including protocol overhead, according to Proposition 2), instead of "the volume of traffic" in bits, occupied by its own transmissions and nearby WSs so far. This is implemented by Algorithm 2 (a revision based on "traffic share estimation algorithm" [9]). According to the measurement results, it dynamically adjust its $\mathrm{CW}$ to achieve fair share of transmission time ("Backoff window adjustment algorithm" [9]). Alternatively, it could adjust the length of its TXOP instead of CWMin in a similar way (not listed in this paper due to the space limit).

For convenient presentation, we cite the global version of Algorithm 1, which compute the max-min fair share in an ad hoc network, given the flow contention graph. Interested readers are referred to [9] for Algorithm 1 itself. Note that Algorithm 1 and its global version yield the same results.

Our focus in this paper is to evaluate how well Algorithm 1 approximates our objective of proportional fairness. This will be elaborated in Section V.
Global version of Algorithm 1. Max-min fair share allocation in a global contention graph. It returns $S=\left\{s_{1}, s_{2}, \ldots, s_{x}\right\}$ as the fair share of each flow.

Let $C=\left\{c_{1}, c_{2}, \ldots, c_{m}\right\}$ be the capacities of the cliques, all initialized to 1 ;

Let $D=\left\{d_{1}, d_{2}, \ldots, d_{m}\right\}$ be the initial degrees of the cliques;

Define $U C L$ as the set of unfinished cliques, initialized to $C$; 1: Begin

2: While $\max \left\{d_{i}\right\}>1$ do

3: Sort $U C L$ in a non decreasing order of $C D^{-1}$

$$
\frac{c_{i_{1}}}{d_{i_{1}}} \leq \frac{c_{i_{2}}}{d_{i_{2}}} \leq \ldots \leq \frac{c_{i_{n}}}{d_{i_{n}}}
$$

4: Assign a fair share to all vertices in clique $C l_{i_{1}}$ :

$$
s_{v}=\frac{c_{i_{1}}}{d_{i_{1}}}, \text { where } v \in C l_{i_{1}}
$$

5: Remove all vertices of $C l_{i_{1}}$ from all other cliques of $U C L$ and update the degrees and capacities of the cliques

For any $C l_{k} \in U C L$,

$$
\begin{aligned}
& C l_{k}=C l_{k} \backslash C l_{i_{1}} \cap C l_{k} \\
& d_{k}=d_{k}-\left|C l_{i_{1}} \cap C l_{k}\right| \\
& c_{k}=c_{k}-\frac{c_{i_{1}}}{d_{i_{1}}} *\left|C l_{i_{1}} \cap C l_{k}\right|
\end{aligned}
$$

6: End while

Algorithm 2. Air Time share estimation algorithm at each sending node.

Init

Let $s_{e}$ be the aggregate air time fair share of flows $V=\left\{v_{1}, v_{2}, \ldots, v_{n}\right\}$ originating at this node,

Let $s_{o}$ be the aggregate air time fair share of flows $V^{\prime}=\left\{v_{1}^{\prime}, v_{2}^{\prime}, \ldots, v_{l}^{\prime}\right\}$ sensed by this node $V \cap V^{\prime}=\varnothing$

Let $T e$ be the measured air time (including the protocol overhead) of flows in $V$, $\left(T_{D A T A}+T_{\text {overhead }}\right.$ in the following algorithm is the time needed for transmitting a data packet and its overhead. It should be embedded in both DATA and ACK packets)

Let $T o$ be the measured air time of flows in $V$,

Let Last Sender be a variable used to correlate ACKs and DATA packets for the case where both sender and receiver are in $V^{\prime}$, this variable is reset periodically if not changed by the algorithm (the period depends on the protocol (e.g., in 802.11, it should be about SIFS+propagation delay).
1: Begin
2: For each packet $p$
3: if $(p \rightarrow$ Destination Id $==$ My Id $)$ then
4: if $(\mathrm{p} \rightarrow$ Type $==$ ACK $)$ then
5: $T e=T e+T_{D A T A}+T_{\text {overhead }}$
6: else
7: if $(\mathrm{p} \rightarrow$ Type $==$ DATA $)$ then
8: $T o=T o+T_{D A T A}+T_{\text {overhead }}$
9: end if
10 : end if 
11: else

12: if $(p \rightarrow$ Type $==$ ACK $)$ then

13: if ( $\mathrm{p} \rightarrow$ Destination Id $\neq$ Last Sender) then

14: $T o=T o+T_{D A T A}+T_{\text {overhead }}$

15: end if

16: else

17: if $(\mathrm{p} \rightarrow$ Type $==$ DATA $)$ then

18: $T o=T o+T_{D A T A}+T_{\text {overhead }}$

19: Last Sender $=\mathrm{p} \rightarrow$ Sender Id

20: end if

21: end if

22: end if

23: End

\section{Simulation EVALUATION}

\section{A. WLAN}

Our simulation set-up assumes a 802.11a WLAN with eight WSs sending saturated uplink traffic to the AP. The payload size is fixed at 1460 Bytes. Only WS 1 uses a low data rate of $6 \mathrm{Mbps}$ (e.g. because it is far from the AP and the signal is weak, so that it must decrease its data rate to keep connected), while others use $36 \mathrm{Mbps}$ (e.g. they are near to the AP). For "max-min bandwidth fairness", all the CWMin's are the same, and in "proportional bandwidth fairness", CWMin's are adjusted according to (18). The value of CWMin's can be distributed from the AP to its WSs, since the AP knows the individual air-time used by a packet burst from each WS.

Here, we keep all the CWMin above 32, to avoid excessive packet collisions. If CWMin's are too small, excessive collisions will severely degrade the throughput of the WLAN; on the other hand, if CWMin's are too large, there will be unnecessary idle slots. How to optimally choose CWMin's is beyond the scope of this paper. However, since the penalty of too small CWMin's is much larger, we keep all the CWMin's above 32 here. No channel errors are assumed. So, according to (18),

$$
\begin{aligned}
& W_{k}=32, k=2,3, \ldots, 8 \\
& W_{1}=32 * T_{1} / T_{k}=32 * 2143 \mu \mathrm{s} / 467 \mu \mathrm{s} \approx 146
\end{aligned}
$$

Note that adjusting CWs is not the only strategy, and using the same TXOP for each WS is even simpler.

Figure 2 (a) and (b) show the bandwidth/throughput allocation and air-time allocation provided by Max-min fairness and proportional fairness. According to Property 1, in proportional fairness, the reduction of data rate to $6 \mathrm{Mbps}$ by WS 1 does not affect other WSs' throughput. From (b), we see that the settings in (20) effectively allocate air-time fairly to the 8 WSs.

The total throughput/goodput of $8 \mathrm{WSs}$ is about $24 \mathrm{Mbps}$ if all the WSs use the data rate of $36 \mathrm{Mbps}$. When WS 1 reduces its data rate to $6 \mathrm{Mbps}$, to achieve max-min fairness, the total throughput falls to $16.69 \mathrm{Mbps}$, while in proportional fairness (or max-min fairness in the time usage), 22.09 Mbps is achievable.

If there are fewer WSs (.e.g. 2 WSs) in a WLAN, and the data rates differ more significantly (e.g. one uses $1 \mathrm{Mbps}$ while the other $11 \mathrm{Mbps}$ ), the inefficiency of Max-min fairness is more distinct.

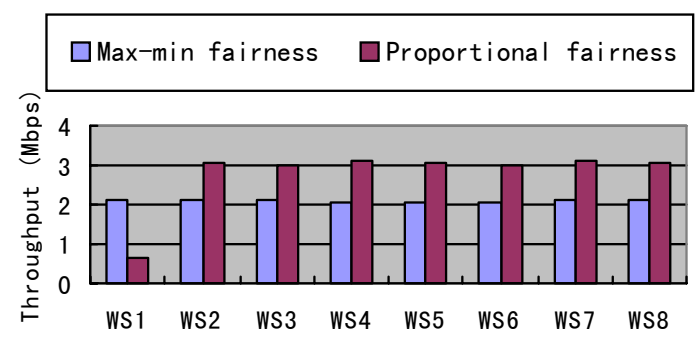

(a) Individual Throughput of 8 WSs (In proportional fairness, WS 1 's low data rate only affect its own throughput)

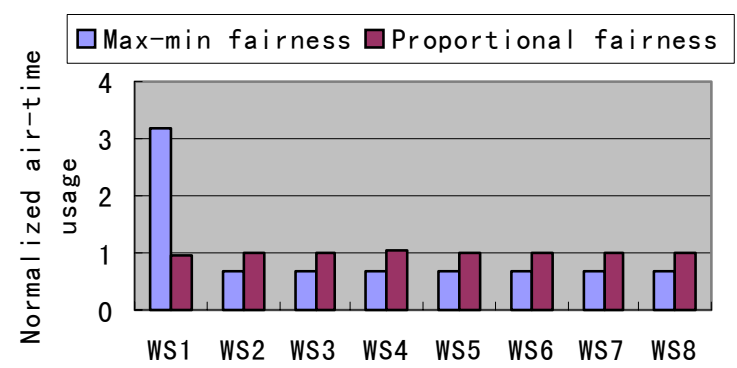

(b) Individual air-time usage of 8 WSs (Normalized)

Figure 2. Comparison of max-min fairness (allocating the same bandwidth/throughput to all WSs) and proportional fairness (allocating the same "air-time" to WSs), when WS 1's data rate is 6Mbps, while others' are $36 \mathrm{Mbps}$

\section{B. Ad hoc Networks}

A WLAN can be regarded as a special case of ad hoc networks. In this sub-section, we allocate the "air-time" in an ad hoc network in a max-min manner, using Algorithm 1 in the distributed Time-Max-Min (TMM) algorithms described in Section IV. The purpose is to find out how close Algorithm 1 approaches ideal proportional fairness. (The effectiveness of Algorithm 2 and 3 for each WS to achieve the target share determined by Algorithm 1 has been evaluated in [9]). To evaluate the approach, we first compute the "normalized" shares, then obtain the "Fairness Index" of them, as follows.

Let $N$ be the number of flows in an ad hoc network, these flows constitute a set $F$, and let the contention graph of this set be $G(F)$. We first solve the optimization problem in (19), and obtain the ideal proportional fairness time share vector $P F[i]$, where $i=1,2, \ldots N$. The actual time share vector we get from the Algorithm 1 in Section IV is TMM[i]. Then, the normalized share of flow $i$

$$
F_{n}[i]=\frac{T M M[i]}{P F[i]}
$$

Finally, the Fairness Index [13] is computed by

$$
F I=\frac{\left(\sum_{i=1}^{N} F_{n}[i]\right)^{2}}{N \sum_{i=1}^{N} F_{n}[i]^{2}}
$$

Example 1: 


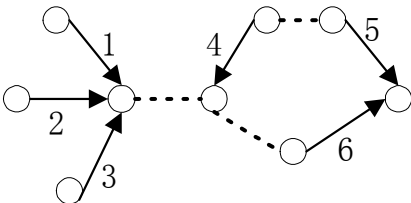

(a) Node graph

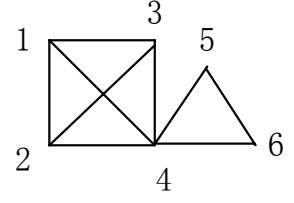

(b) Flow contention graph

Figure 3. Node graph and Flow contention graph of Example 1. $\{1,2,3,4\}$ and $\{4,5,6\}$ are two cliques (the dashed lines in (a) refer to interference)

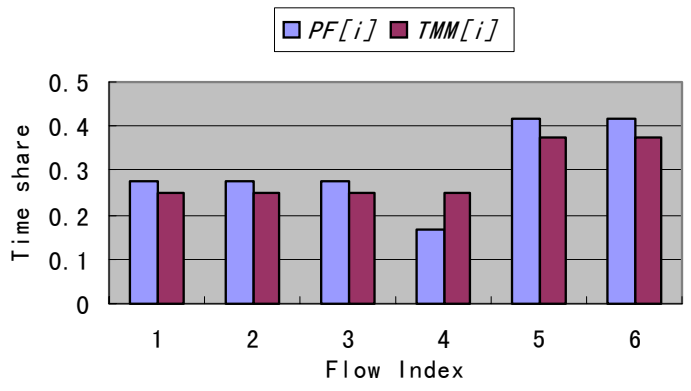

Figure 4. Using max-min time allocation to approximate proportional fairness - Example 1 (Result: Fairness Index $=0.86$ )

Example 2:
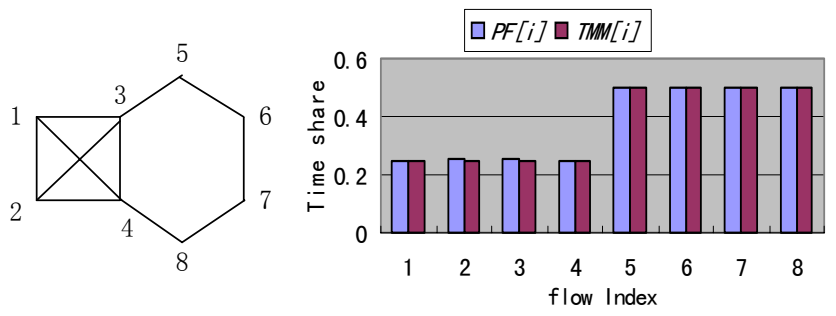

(a) Flow contention graph

(b) Comparison of $P F[i]$ and $T M M[i]$

Figure 5. Using max-min time allocation to approximate proportional fairness - example 2 (Result: Fairness Index $=1.00$ )

In Fig 3, for example, $\{1,2,3,4\}$ and $\{4,5,6\}$ are two cliques. Using Algorithm 1, flow 1, 2, 3, 4 get an air-time share of $1 / 4$ in the first iteration, and flow 5,6 get a share of $3 / 8$ in the second iteration. In the evaluation given above $(F I=0.86$ in Example 1 and $F I=1.00$ in Example 2), local max-min time allocation yields a reasonably good approximation to global proportional fairness.

\section{CONCLUSION}

In wireless LANs and ad hoc networks, fairness and efficiency can be two conflicting objectives, particularly so in a multi-rate environment in which wireless stations may use different data transmission rates. To strike a balance between the two objectives, a popular performance objective is the proportional fairness objective. It turns out that proportional fairness in bandwidth usage (which does not have much of a physical meaning except that it is a convenient mathematical objective function used as a compromise between the overall network throughput and fairness to individual nodes) does have a physical meaning in the multi-rate environment in terms of air-time usage.

In particular, we have proved the analytical equivalence of proportional fairness objective and the max-min airtime usage objective in WLANs. By allocating air-time to wireless stations in a fair manner, proportional fairness in bandwidth usage is achieved. Based on this insight, we have investigated two approaches to achieving proportional fairness by means of airtime usage tracking.

In ad hoc networks, achieving proportional fairness in the strict sense can be formulated as a global optimization problem using a flow contention graph. For this optimization, each WS needs global information of flow contention to compute its fair share of air-time, making the task more challenging than in infrastructure WLANs in which AP is the coordinator. A good approximation is for the distributed nodes to monitor only the air-time usage in its surrounding neighborhood. After all, a node contends for air-time usage only with the neighbors that it can hear. In this paper, we have investigated a set of distributed max-min air-time allocation algorithm, referred to collectively as $T M M$ to meet this goal.

\section{REFERENCE}

[1] IEEE Standards Department, IEEE 802.11 Standard for Wireless LAN, Medium Access Control (MAC) and Physical Layer (PHY) Specifications, 2003.

[2] P. Gupta, P. R. Kumar, "The Capacity of Wireless Network", IEEE Trans. on Information Theory, Vol. 46, No. 2, pp.388-404, Mar. 2000.

[3] IEEE Std 802.11g-2003 (Amendment to IEEE Std 802.11, 1999 Edn. (Reaff 2003) as amended by IEEE Stds 802.11a-1999, 802.11b-1999, 802.11b-1999/Cor 1-2001, and 802.11d-2001)

[4] M. Heusse, F. Rousseau, G. Berger-Sabbatel, and A. Duda, "Performance anomaly of 802.11b," IEEE Infocom'03, San Francisco, USA, Mar. 2003.

[5] C. H. Nam, "A Study of Throughput Performance in $802.11 \mathrm{~b}$ Wireless LAN," MPhil Thesis, The Chinese University of Hong Kong, July 2003

[6] F. P. Kelly, "Charging and rate control for elastic traffic," European Transactions on Telecommunications, volume 8 (1997), pp 33-3.

[7] Boj¡zidar Radunovi'c and Jean-Yves Le Boudec, "Rate Performance Objectives of Multi-hop Wireless Networks," INFOCOM'04, Hong Kong

[8] Godfrey Tan, John Guttag, "Time-based Fairness Improves Performance in Multi-rate Wireless LANs," The USENIX Annual Technical Conference, Boston, MA, June 2004.

[9] X. L. Huang, B. Bensaou, "On Max-min Fairness and Scheduling in Wireless Ad-Hoc Networks: Analytical Framework and Implementation", ACM MobiHoc'01, pp. 221-231, Oct. 2001

[10] Bianchi, G., "Performance analysis of the IEEE 802.11 distributed coordination function," Selected Areas in Communications, IEEE Journal on, Volume: 18 Issue: 3, March 2000, pp. 535 -547

[11] IEEE 802.11e/D4.0, Draft Supplement to Part 11: Wireless Medium Access Control (MAC) and physical layer (PHY) specifications: Medium Access Control (MAC) Enhancements for Quality of Service (QoS), November 2002.

[12] Ping Chung Ng, Soung Chang Liew, and Li Bin Jiang, "A Performance Evaluation Framework for IEEE 802.11 Ad-hoc Networks," to appear in ACM Workshop on Performance Evaluation of Wireless Ad Hoc, Sensor, and Ubiquitous Networks (PE-WASUN' 2004).

[13] R. Jain, D. Chiu, W. Hawe, "A Quantitative Measure of Fairness and Discrimination for Resource Allocation in Shared Computer Systems", DEC Report, DEC-TR-301, September 1984. 